\title{
Validação do Questionário Crenças acerca da Medicação em Pacientes Diabéticos Tipo 2
}

\author{
Maria da Graça Pereira ${ }^{1}$ \\ Susana Pedras \\ J. Cunha Machado \\ Universidade do Minho
}

\begin{abstract}
RESUMO - O presente trabalho teve como objectivo validar o Questionário Crenças sobre a Medicação, que avalia Crenças Gerais e Crenças Específicas, estudando suas propriedades psicométricas em uma amostra de 387 pacientes diabéticos tipo 2. O estudo de validade para as Crenças Gerais revelou uma solução de um factor, com um alfa de 0,76, e para as Crenças Específicas, dois factores - Necessidades e Preocupações -, com um alfa de 0,77 e 0,69 respectivamente. Quanto à validade de constructo, verificou-se uma relação entre as Crenças Gerais e a subescala Necessidades das Crenças Específicas com Adesão à Medicação, avaliada pela Escala de Avaliação de Aderência Médica. O instrumento apresenta boas qualidades psicométricas para ser utilizado em pacientes diabéticos tipo 2 .
\end{abstract}

Palavras-chave: crenças, medicação, adesão, diabetes tipo 2

\section{Validation of the Questionnaire on Beliefs about Medication with Type 2 Diabetic Patients}

\begin{abstract}
The present paper focused on the validation of the Questionnaire on Beliefs about Medication, which assesses both General Beliefs and Specific Beliefs. The psychometric properties of the instrument were analyzed on a sample of 387 type 2 diabetic patients. The validity study for General Beliefs found a unifactorial solution, with an alpha of .76, and for Specific Beliefs, a two-factor solution - Necessities and Concern -, with an alpha of .77 and .69, respectively. In terms of construct validity, a relationship between General Beliefs, subscale Necessities from Specific Beliefs, and adherence to medication, as evaluated by Medical Adherence Rating Scale, was found. The instrument presents good psychometric qualities to be used in type 2 diabetic patients.
\end{abstract}

Keywords: beliefs, medication, adherence, diabetes type 2

No confronto com a doença, a forma como o indivíduo reage aos sintomas físicos é influenciada pela sua visão pessoal de saúde e de doença (Reis, 2005). Assim, a forma como as pessoas representam a sua doença pode afectar o modo como lida com ela. O indivíduo é um agente ativo na escolha dos cuidados de saúde e nas decisões sobre o seu próprio tratamento. A partir das crenças pessoais dos indivíduos é possível predizer diferentes comportamentos relacionados com a saúde, tanto no que diz respeito à prevenção da doença quanto à promoção da saúde (Teixeira \& Leal, 1993).

Os estudos demonstram uma fraca relação entre as variáveis clínicas e demográficas e a adesão à medicação (Horne \& Weinman, 1999; Phatak \& Thomas, 2006; Vermeire, Hearnshaw, Van Royen, \& Denekens, 2001), mas referenciam as crenças acerca da medicação como um dos factores que mais afecta a adesão terapêutica (Britten, 1994; Horne, 1997; Horne \& Weinman, 1999). As crenças acerca dos fármacos podem influenciar os comportamentos relacionados com a saúde (Figueiras et al., 2009), como a procura dum tratamento específico, bem como a adesão terapêutica que implica uma série de comportamentos como alterar hábitos de vida ou cumprir uma dieta que coincida com o regime terapêutico

1 Endereço para correspondência: Universidade do Minho, Escola de Psicologia, Campus de Gualtar, 4710-057, Braga, Portugal.

E-mail: gracep@psi.uminho.pt prescrito (World Health Organization, 2003). A perspectiva do paciente relativamente às suas convicções pessoais sobre os medicamentos tem uma importância fulcral na adesão à medicação. As crenças sobre o tratamento desenvolvem-se de acordo com os modelos de senso comum sobre a doença, e com as expectativas relativamente à eficácia e necessidade desse tratamento (Horne, 2001a). Para Horne (2006), as crenças sobre o tratamento em geral e, sobre os medicamentos em particular, são influenciadas pelas crenças sobre a doença e, sobretudo pela percepção da sua gravidade. Segundo vários estudos, o modelo da necessidade-preocupação é útil para perceber e explicar a não adesão (Aikens, Nease, Nau, Klinkman, \& Schwenk, 2005; Aikens \& Piette, 2009; Butler et al., 2004; Horne, Frost, Hankins, \& Wright, 2001; Horne \& Weinman, 1999; Neame \& Hammond, 2005; Ross, Walker, \& MacLeod, 2004). As preocupações com a medicação podem traduzir uma reacção emocional de medo e ansiedade provocando uma diminuição da adesão ou influenciando a escolha do medicamento (Horne, 2003). As crenças sobre a medicação, e a forma como os indivíduos equilibram as suas preocupações em relação aos efeitos da medicação com a percepção da sua necessidade, são preditores da adesão à medicação em várias doenças crónicas (Butler et al., 2004; Horne, 1997; Horne, Weinman, \& Hankins, 1999; Horne \& Weinman, 2002; Neame \& Hammond, 2005). 
Horne et al. (1999) verificaram em pacientes portadores de diversas doenças, entre elas a diabetes, que os pacientes com crenças acerca da necessidade do tratamento apresentavam taxas mais elevadas de adesão. A combinação de crenças positivas e negativas acerca da medicação são preditores muito mais poderosos do que os factores sociodemográficos ou clínicos (Horne \& Weinman, 1999). A visão negativa sobre a medicação, as crenças associadas à noção de que os aspectos perigosos da medicação estão associados às origens químicas e artificiais, a percepção de serem substâncias nocivas utilizadas em demasia pelos médicos, a percepção de que os tratamentos complementares são mais "naturais" e mais seguros (Horne et al., 1999), podem influenciar as preferências de tratamento e até o curso dos cuidados de saúde (Horne, 1999). Neame e Hammond (2005) encontraram uma correlação positiva entre a necessidade específica de medicação e as preocupações específicas com a medicação. Britten (1994), tal como Treharne, Lyons e Kitas (2004), referem que as convicções fortes sobre a necessidade de medicamentos e a crença que os medicamentos geralmente não vão ser usados por muito tempo são preditores de uma maior adesão terapêutica. A não adesão está frequentemente relacionada com o modo como os pacientes avaliam a necessidade do medicamento $\mathrm{e}$, nesse caso, a não adesão pode ser devida às preocupações sobre os efeitos negativos da toma da medicação (Horne, 2006; Treharne et al., 2004). De facto, estudos sobre crenças na diabetes revelam que os pacientes que apresentam mais crenças sobre a necessidade e menos crenças de preocupação da medicação aderem mais (Barnes, Moss-Morris, \& Kaufusi, 2004; Farmer, Kinmonth, \& Sutton, 2006; McHorney \& Gadkari, 2010; Nair, Levine, Lohfeld, $\&$ Gerstein, 2007).

O processo de adesão é um processo de tomada de decisão racional, na qual os pacientes equilibram as suas preocupações acerca da medicação com a percepção da sua necessidade e a percepção de benefícios (Benson \& Britten, 2002; Donovan \& Blake, 1992; Dowell \& Hudson, 1997; Elliott, Ross-Degnan, Adams, Safran, \& Soumerai, 2007; Lau et al., 2008; Nair et al., 2007). As crenças dos pacientes acerca do tratamento, sua condição e prognóstico, bem como as suas experiências objectivas com o tratamento, diferenciam os pacientes aderentes dos não aderentes (Aikens et al., 2005; Byrne, Walsh, \& Murphy, 2005; Clifford, Barber, \& Horne, 2008; Horne et al., 2004; Menckeberg et al., 2008; Phatak \& Thomas, 2006). Outro aspecto a ter em consideração é o facto de os pacientes apresentarem diferentes padrões de adesão perante diferentes medicações. As suas decisões são tomadas tendo em conta as crenças associadas a determinada medicação, conhecimento acerca dessa medicação e a condição médica em causa. Sendo assim, existem pacientes que são não aderentes a determinada medicação, mas são aderentes a outra porque tomam decisões separadas para cada uma delas (McHorney \& Gadkari, 2010). Aliás, os pacientes apresentam uma valorização distinta e têm diversas crenças perante diferentes medicações tendo em conta a sua percepção de importância, eficácia, segurança e benefícios esperados (Aikens \& Piette, 2009; Ayalon, Arean, \& Alvidrez, 2005;
Chapman, Petrilla, Benner, Schwartz, \& Tang, 2008; Elliott et al., 2007; Williams, Manias, \& Walker, 2008). Assim, os pacientes medicados com ambos antidiabéticos orais e anti-hipertensivos, aderem ao medicamento classificado como o mais importante e necessário (Aikens \& Piette, 2009; McHorney \& Gadkari, 2010).

Em vários estudos com doença crónica foi encontrada uma relação entre as crenças gerais acerca da medicação e a adesão em pacientes (Farmer et al., 2006; Horne et al., 1999; Nair et al., 2007; Neame \& Hammond, 2005). Assim, as crenças mais fortes acerca da medicação percebidas como prejudiciais estão associadas a menor adesão terapêutica (Mardby, Akerlind, \& Jorgensen, 2007).

A incidência da diabetes tem aumentado na população mundial sendo considerada, por isso, uma das maiores causas de morbilidade e mortalidade na actualidade (Duarte, 2004). Os estudos indicam que a não adesão ao tratamento é um dos principais problemas da diabetes (Asefzadeh, Asefzadeh, \& Javadi, 2005). A diabetes tipo 2, em particular, é uma das doenças crónicas em que a adesão terapêutica é fundamental, pois as complicações que podem resultar de uma má adesão terapêutica implicam custos elevados para o paciente e para a sociedade estimando-se que a percentagem de pacientes que não adere a medicação oral varia entre 13\% a 64\% (Cramer, 2004; Lee, Balu, Cobden, Joshi, \& Pashos, 2006).

Dada a taxa elevada da não adesão em diabéticos tipo 2 e a importância das crenças acerca dos fármacos na adesão terapêutica, decidimos estudar as propriedades psicométricas do questionário de crenças acerca dos fármacos em diabéticos tipo 2. De facto, em Portugal, não existe nenhum instrumento de avaliação de crenças acerca dos fármacos adaptado a doentes crónicos, apenas um instrumento que avalia as representações da doença a nível cognitivo e emocional e que se insere no modelo teórico de Leventhal, Meyer e Nerenz (1980) que revelou que as crenças dos pacientes acerca da doença eram fundamentais para perceber a forma como os doentes escolhem lidar com a sua doença. Em particular, na diabetes, verificou-se, que os pacientes que acreditam ter maior controle sobre a doença procuram mais os serviços de saúde e apresentam mais comportamentos de saúde (Hampson, Glasgow, \& Foster, 1995; Kavanagh, Gooley, \& Wilson, 1993).

Nessa linha de pensamento, o presente estudo teve como objectivo a validação do Questionário Crenças acerca da Medicação (Beliefs about Medicines Questionnaire - BMQ, Horne et al.,1999) em pacientes diabéticos tipo 2. De acordo com a literatura, existe uma relação negativa entre as crenças acerca dos fármacos e a adesão terapêutica. Pacientes diabéticos que acreditam nos efeitos nocivos e uso excessivo dos medicamentos (crenças gerais), bem como possuem mais preocupações em relação á medicação (crenças especificas), apresentarão menor adesão terapêutica. Neste sentido, foram investigadas as relações entre crenças sobre medicação e o comportamento de adesão à medicação no que diz respeito à validade convergente. Quanto à validade discriminante, foram avaliadas as diferenças entre homens e mulheres, bem como entre pacientes mais idosos e jovens. 


\section{Método}

\section{Participantes}

No estudo participaram 387 pacientes diabéticos (58\% homens e $42 \%$ mulheres) diagnosticados com diabetes tipo 2. A média de idades foi de 59 anos $(\mathrm{DP}=10,4)$, sendo que o mais novo do participante tinha 30 anos e o mais velho 86 anos. Na amostra, $67 \%$ apresentava quatro anos de escolaridade e $14,7 \%$ seis anos de escolaridade (ensino fundamental). Relativamente à duração do diagnóstico, $57 \%$ dos pacientes recebeu o diagnóstico seis meses anterior à avaliação e $38 \%$ entre sete e 12 meses. Todos os pacientes encontravam-se em uso de medicação oral.

\section{Instrumentos}

O Questionário Crenças Acerca da Medicação (BMQ, Horne et al., 1999) foi desenvolvido para avaliar as crenças que os indivíduos possuem em relação aos fármacos, i.e., avalia as crenças comuns que as pessoas possuem sobre a medicação. A versão original é composta por duas partes, designadas por: Parte I - Crenças Gerais sobre a Medicação (oito itens: BMQ - Crenças Gerais) e Parte II - Crenças Específicas sobre a Medicação (10 itens: BMQ - Crenças Específicas). A primeira parte avalia crenças gerais acerca dos medicamentos, enquanto a segunda avalia as crenças específicas em relação aos fármacos prescritos, para uso pessoal, ao doente. A Parte I (Escala de Crenças Gerais) é constituída por dois factores de quatro itens. O primeiro factor, Escala Efeitos Nocivos, avalia crenças relacionadas com potenciais efeitos nocivos, aditivos e tóxicos dos medicamentos, é composta por quatro itens (item 2, 3, 5, 6) e apresenta um alfa de 0,66. Exemplo dos itens: "Os medicamentos fazem mais mal que bem", "Todos os medicamentos são tóxicos" e "Os produtos naturais são mais seguros que os medicamentos". O segundo factor, Escala de Uso Excessivo, avalia a crença da medicação estar a ser utilizada em excesso pelos médicos, e é composta por quatro itens (item 1, 4, 7,8) e apresenta um alfa de 0,80. Exemplo dos itens: "Os médicos usam medicamentos a mais", "Se os médicos estivessem mais tempo com os doentes, receitariam menos medicamentos" e "Os médicos usam medicamentos a mais".

A Parte II (Escala Crenças Específicas) é composta por dois factores de cinco itens. O primeiro factor, Escala Necessidades da Medicação, avalia crenças acerca da necessidade da medicação que lhes foi prescrita, e é composta por cinco itens (itens 1, 3, 4, 7, 10) e apresenta um alfa de 0,74. Exemplo dos itens: "Sem medicamentos eu estaria muito doente", Neste momento a minha saúde depende de medicamentos" e "A minha saúde no futuro irá depender de medicamentos". O segundo factor, Escala de Preocupações Especificas, avalia crenças acerca dos perigos de dependência e toxicidade ou efeitos secundários a longo prazo, é composta por cinco itens (itens 2, 5, 6, 8, 9) e apresenta um alfa de 0,80 (Horne et al., 1999). Exemplo dos itens:
"Ter que tomar medicamentos preocupa-me", "Às vezes eu preocupo-me com a possibilidade de ficar muito dependente dos meus medicamentos" e "Os meus medicamentos desorganizam a minha vida".

Essas duas partes do BMQ podem ser usadas em combinação ou separadamente consoante os objectivos do estudo. Os participantes respondem indicando o grau de concordância com as afirmações acerca da medicação que varia, segundo uma escala de Likert de cinco pontos ( $1=$ Discordo Totalmente, $2=$ Discordo, $3=$ Não tenho a certeza, $4=$ Concordo e $5=$ Concordo Totalmente). O total na Escala de Efeitos Nocivos varia de 5 a 25; na Escala de Uso Excessivo varia de 5 a 15; na Escala Necessidades Específicas varia de 5 a 25 e na Escala de Preocupações Específicas varia de 5 a 25.

Um resultado elevado em ambas as partes indica crenças gerais e específicas mais fortes sobre a medicação. Assim, a parte geral, indica crenças sobre os efeitos nocivos e uso excessivo da medicação, enquanto que a parte específica indica crenças sobre a necessidade da medicação e as preocupações com a medicação.

Em Portugal, em doentes com lombalgia, Ferreira (2009), encontrou alfas de 0,73 e 0,77 para a Parte I (Crenças Gerais: Uso Excessivo e Toxicidade) e 0,74 para a Parte II (Crenças Específicas), não tendo sido encontrada a solução de quatro factores. Vários estudos internacionais têm sido desenvolvidos com esse instrumento. Aikens e Piette (2009) e Barnes et al. (2004) utilizaram apenas a Parte II (Crenças Específicas) em amostras de diabéticos avaliando respectivamente as crenças em relação aos antiglicémicos e anti-hipertensivos e a relação entre as crenças e a não adesão e confirmaram as duas subescalas originais. Nesse último estudo, verificou-se também, que crenças acerca da medicação estavam associadas a não adesão à dieta e à medicação. Ramston, Afandi, Elofsson e Petersson (2009) utilizaram apenas a Parte I (Crenças Gerais) em uma amostra de pacientes com doença crónica confirmando as duas escalas originais e verificando que as crenças sobre os efeitos nocivos estavam associadas à não adesão. Por sua vez, Tibaldi et al. (2009) utilizaram a Parte II (Crenças Específicas) em pacientes com asma, diabetes, depressão e doença cardíaca e confirmaram as duas subescalas. Finalmente Tordera, Moragon, Fuster, Bayo e Ciscar (2009) utilizaram as Partes I e II e confirmaram a existência de quatro factores em pacientes com asma.

A Escala de Avaliação de Aderência Médica (Medication Adherence Report Scale - MARS, Horne, 2001b) tem por objectivo avaliar os níveis de adesão à medicação, nomeadamente a frequência com que os sujeitos ajustam ou alteram as doses prescritas pelo médico. A MARS é composta por cinco itens. A escala tem uma pontuação máxima de 25 pontos, resultante da soma do somatório de todos os itens (Horne, 2001b). Resultados elevados indicam maiores níveis de adesão. Esse instrumento possui propriedades psicométricas razoáveis quanto à fidelidade, apresentando um alfa de 0,70 na versão original e de 0,74 em uma versão portuguesa adaptada em diabéticos tipo 2. Exemplos de itens: "Eu esqueço-me de tomar a medicação", "Eu altero a dose" e "Eu paro de os tomar durante algum tempo". 


\section{Procedimentos}

A amostra foi recolhida em postos de saúde do Norte de Portugal onde os pacientes recebem cuidados de saúde de rotina para a diabetes, de forma gratuita. Os pacientes diabéticos têm assim acesso a quatro consultas anuais com um médico(a) e enfermeiro(a), que o Serviço Nacional de Saúde, oferece em todas as zonas do país, dado a diabetes ser uma doença crónica de elevado risco para o paciente e custos para a saúde. O estudo teve a duração de três anos e foi aprovado pela Comité de Ética da Associação Regional de Saúde (ARS) da Zona Norte de Portugal. Todos os participantes eram conhecedores do objectivo do estudo e assinaram um consentimento informado. A participação foi voluntária e todos os pacientes foram convidados a participar no estudo pelo seu médico de família desde que preenchessem os critérios de inclusão, na amostra. Os dois instrumentos foram preenchidos sequencialmente na presença de um psicólogo que integrava a equipe de investigação. Foram excluídos os pacientes diabéticos portadores de doença oncológica. Os critérios de inclusão incluíram diagnóstico de diabetes até um ano no momento da avaliação, mais de 18 anos e tomar apenas com medicação oral para a diabetes. Os doentes eram avaliados no primeiro mês após o diagnóstico.

O processo de tradução e adaptação teve como base a orientação proposta por Bradley (1996) e decorreu de acordo com o seguinte procedimento: tradução pelo investigador e simultaneamente por tradutor independente; confronto das versões para elaboração da primeira versão em português; retroversão por tradutor independente, não conhecedor da versão inicial em língua inglesa; confronto de versões (original e retrovertida), com o objectivo de avaliar a identidade do conteúdo dos itens e adaptação e correção dos termos técnicos por um médico; pré-teste com 10 diabéticos para avaliar a adequação e compreensão dos itens da versão de investigação e finalmente a elaboração da versão a utilizar no presente estudo. Os diabéticos que integraram o pré-teste foram recolhidos nos mesmos postos de saúde da amostra e foi realizada, com cada paciente, uma reflexão falada acerca das potenciais dúvidas nos itens. Desse processo, foi necessário introduzir pequenas modificações apenas no questionário sociodemográfico.

\section{Resultados}

Em relação às propriedades psicométricas do Questionário Crenças acerca da Medicação (BMQ), o estudo da validade incluiu a análise factorial em componentes principais, com rotação ortogonal através do método varimax. Dado que os autores originais sugerem a independência do BMQ - Geral versus BMQ - Específico e pelo facto de vários estudos, já anteriormente referidos, incluírem apenas um desses dois instrumentos, o estudo de validade foi realizado para a Parte I e a Parte II separadamente. A adequabilidade da análise factorial aos itens, através da verificação da existência de correlações significativas entre eles, foi confirmada pela medida de Kaiser-Meyer-Olkin $(\mathrm{KMO}=0,749)$ e pelo teste de esfericidade de Bartlett $(\mathrm{QQ}=678,3 ; \mathrm{gl}=28 ; \mathrm{p}<0,01)$.
Tabela 1. Análise Factorial e Valor de Alfa da Parte I: Crenças Gerais sobre a Medicação nos Pacientes Diabéticos (8 Itens)

\begin{tabular}{lcc}
\hline Itens & Saturação & $\begin{array}{c}\text { Correlação } \\
\text { item total }\end{array}$ \\
\hline $\begin{array}{l}\text { 7 - Os médicos confiam demais } \\
\text { nos medicamentos }\end{array}$ & 0,776 & 0,618 \\
$\begin{array}{l}8 \text { - Se os médicos estivessem } \\
\text { mais tempo com os doentes, re- } \\
\text { ceitariam menos medicamentos }\end{array}$ & 0,676 & 0,507 \\
$\begin{array}{l}\text { 1 - Os médicos usam medica- } \\
\text { mentos a mais }\end{array}$ & 0,673 & 0,508 \\
$\begin{array}{l}5 \text { - Os medicamentos fazem } \\
\text { mais mal que bem }\end{array}$ & 0,631 & 0,488 \\
$\begin{array}{l}\text { 6 - Todos os medicamentos são } \\
\text { tóxicos }\end{array}$ & 0,599 & 0,422 \\
$\begin{array}{l}\text { - Os produtos naturais são } \\
\text { mais seguros que os medica- } \\
\text { mentos }\end{array}$ & 0,572 & 0,428 \\
$\begin{array}{l}\text { 3 - Muitos medicamentos pro- } \\
\text { vocam dependência }\end{array}$ & & \\
$\begin{array}{l}\text { 2 - Pessoas que tomam medi- } \\
\text { camentos deveriam parar o seu } \\
\text { tratamento durante um certo } \\
\text { tempo de vez em quando }\end{array}$ & 0,520 & 0,385 \\
\hline
\end{tabular}

Nota . Valor próprio $=3,029$; Variância explicada $=37,87 \%$; Alpha de Cronbach $=0,76$.

Os resultados obtidos na Parte I, Crenças Gerais, que na versão original está dividida em duas subescalas - Uso Excessivo e Efeitos Nocivos - revelaram que a melhor solução inclui apenas um factor que explica $37,87 \%$ da variância. Os alfas encontrado na Parte I, constituída por oito itens, foi de 0,76 , e as correlações entre os itens oscilam entre 0,28 e 0,61. A Tabela 1 apresenta os resultados.

O estudo da validade da Parte II, Crenças Específicas, que na versão original está dividida em duas subescalas Necessidades Especificas e Preocupações Especificas - foi levado a cabo através da análise factorial em componentes principais, com rotação ortogonal através do método varimax. A adequabilidade da análise factorial aos itens, através da verificação da existência de correlações significativas entre eles, foi confirmada pela medida de Kaiser-Meyer-Olkin $(\mathrm{KMO}=0,722)$ e pelo teste de esfericidade de Bartlett $(\mathrm{QQ}=$ 895,98; $\mathrm{gl}=45 ; \mathrm{p}<0,01)$. Os resultados obtidos revelaram que a melhor solução incluía dois factores, tal como o autor sugere na versão original. Um factor explica $26,85 \%$ e outro $22,44 \%$ da variância total dos resultados. O primeiro factor inclui todos os itens 1, 3, 4, 7, 10 da subescala Crenças Necessidades Específicas e o segundo factor inclui todos os itens 2, 5, 6, 8, 9 pertencentes à subescala Crenças Preocupações Específicas. Na Parte II, Crenças Específicas, foram então encontrados dois factores tal como o autor sugeriu. $\mathrm{O}$ valor de alfa encontrado para a subescala Crenças sobre as Necessidades Específicas foi de 0,77 e as correlações entre os itens e a escala oscilam entre 0,46 e 0,68. Na subescala 
Tabela 2. Análise Factorial e Fidelidade da Parte II Subescala: Crenças Necessidade Especificas nos Pacientes Diabéticos (5 Itens)

\begin{tabular}{lcc}
\hline Itens & Saturação & $\begin{array}{c}\text { Correlação do } \\
\text { item com o total }\end{array}$ \\
\hline $\begin{array}{l}\text { 4 - Sem medicamen- } \\
\text { tos eu estaria muito } \\
\text { doente }\end{array}$ & 0,818 & 0,676 \\
$\begin{array}{l}\text { 1 - Neste momento a } \\
\text { minha saúde depende } \\
\text { de medicamentos }\end{array}$ & 0,741 & 0,567 \\
$\begin{array}{l}\text { - A minha saúde no } \\
\text { futuro irá depender } \\
\text { de medicamentos }\end{array}$ & 0,730 & 0,545 \\
$\begin{array}{l}\text { - A minha vida } \\
\text { seria impossível sem } \\
\text { medicamentos }\end{array}$ & 0,687 & \\
$\begin{array}{l}10 \text { - Os meus medica- } \\
\text { mentos impedem-me } \\
\text { de piorar }\end{array}$ & 0,651 & 0,503 \\
\hline
\end{tabular}

Nota . Valor próprio $=2,685$; Variância explicada $=26,85 \%$; Alpha de Cronbach $=0,77$

Crenças sobre as Preocupações Específicas, o alfa encontrado foi de 0,69 e as correlações entre os itens e a escala oscilam entre 0,26 e 0,53 . As Tabelas 2 e 3 apresentam os resultados.

Ainda no que diz respeito à validade de constructo, verificou-se uma relação entre as Crenças Gerais e Crenças Específicas (Necessidade da Medicação) com a Adesão à Medicação como seria de esperar. Não foram encontradas relações entre Crenças Específicas (Preocupação com a Medicação) e Adesão à Medicação (ver Tabela 4).

Tabela 3. Análise Factorial e Fidelidade da Parte II Subescala "Crenças Preocupações Específicas" nos Pacientes Diabéticos (5 Itens)

\begin{tabular}{lcc}
\hline Itens & Saturação & $\begin{array}{c}\text { Correlação } \\
\text { item total }\end{array}$ \\
\hline $\begin{array}{l}\text { 9 - Às vezes eu preocupo-me } \\
\text { com a possibilidade de ficar } \\
\text { muito dependente dos meus } \\
\text { medicamentos }\end{array}$ & 0,759 & 0,529 \\
$\begin{array}{l}5 \text { - Às vezes eu preocupo-me } \\
\text { com os efeitos que os meus me- } \\
\text { dicamentos me podem provocar }\end{array}$ & 0,756 & 0,539 \\
$\begin{array}{l}\text { se eu os tomar durante muito } \\
\text { tempo }\end{array}$ & \\
$\begin{array}{l}\text { 8 - Os meus medicamentos } \\
\text { desorganizam a minha vida }\end{array}$ & 0,682 & 0,491 \\
$\begin{array}{l}\text { - Ter que tomar medicamen- } \\
\text { tos preocupa-me }\end{array}$ & 0,625 & 0,431 \\
$\begin{array}{l}6 \text { - Os meus medicamentos são } \\
\text { um mistério para mim }\end{array}$ & 0,424 & 0,255 \\
\hline
\end{tabular}

Nota . Valor próprio $=2,244$; Variância explicada $=22,44 \% ;$ Alpha de Cronbach $=0,69$
Tabela 4. Relação entre Crenças acerca dos Fármacos (BMQ) e Adesão à Medicação (MARS)

\begin{tabular}{lcc}
\hline & \multicolumn{2}{c}{ MARS } \\
\cline { 2 - 3 } & $r$ & $p$ \\
\hline Crenças Gerais sobre a Medicação & $-0,133$ & 0,009 \\
Crenças Específicas: Necessidade & 0,263 & 0,000 \\
Crenças Específicas: Preocupação & $-0,039$ & 0,445 \\
\hline
\end{tabular}

Quanto à validade discriminante, quando relacionadas as três escalas sobre Crenças acerca da Medicação com a Escala de Adesão à Medicação tendo em consideração o género e a idade, verificaram-se relações significativas. Assim, as mulheres que aderem à medicação apresentam maior número de crenças sobre as necessidades específicas $(r=0,314 ; \mathrm{p}<0,01)$. Já os diabéticos do sexo masculino que aderem à medicação apresentam menor número de crenças gerais (toxicidade $\mathrm{e}$ uso excessivo) acerca da medicação $(r=-0,164 ; \mathrm{p}=0,014)$ embora apresentem mais crenças sobre as necessidades da medicação $(r=0,230 ; p=0,001)$. Relativamente à idade, tanto os diabéticos mais novos (com menos de 60 anos) ( $\mathrm{r}=0,261$, $\mathrm{p}<0,01)$ quanto os mais velhos (mais de 60 anos) ( $\mathrm{r}=0,265$, $\mathrm{p}<0,01)$ que aderem à medicação preocupam-se com as necessidades específicas da medicação.

Em relação ao género, verificamos diferenças significativas nas duas subescalas de Crenças Específicas sobre a Medicação na subescala Necessidades Específicas ( $\mathrm{t}=2,535$, $\mathrm{p}=0,012)$ e na subescala Preocupações Específicas ( $t=3,597$, $\mathrm{p}<0,01)$. Assim, as mulheres apresentam mais crenças sobre necessidades da medicação e sobre preocupações com a medicação quando comparadas com os homens. Contudo, não verificamos diferenças significativas em função da idade quando comparamos mulheres com homens diabéticos.

\section{Discussão}

Neste estudo verificamos que existe uma relação entre as crenças sobre a medicação e a adesão à medicação, o que vem ao encontro de estudos anteriores que apontam as crenças dos pacientes sobre a medicação como um dos factores responsáveis pela adesão terapêutica (Britten, 1994; Horne, 2003, Horne et al., 1999; Horne \& Weinman, 1999). Além disso, encontramos um factor na Parte I (Crenças Gerais), o que difere da versão do autor que refere a existência de duas subescalas: efeitos nocivos (toxicidade) e uso excessivo da medicação. Embora no nosso estudo, a presença dessas duas escalas não se tenham confirmado, os itens mantêm-se os mesmos das subescalas originais. Em relação à Parte II (Crenças Específicas) foram encontrados dois factores tal como o autor original sugere.

As crenças gerais sobre a medicação, tal como seria de esperar, relacionam-se negativamente com a adesão à medicação sugerindo que os diabéticos que possuem menos crenças gerais acerca da medicação (toxicidade e uso excessivo) aderem mais à medicação. Segundo Horne (2003), o medo, os receios e a ansiedade em tomar a medicação relacionadas com a percepção de um uso excessivo e com a toxicidade dos medicamentos podem levar a uma diminuição da adesão 
e influenciar até a escolha do medicamento. Por sua vez, a subescala necessidades específicas acerca da medicação relaciona-se positivamente com a adesão à medicação, o que é compreensível uma vez que se a percepção de necessidade da medicação for elevada, pode superar o medo dos efeitos secundários desagradáveis (Horne et al., 1999; Horne \& Weinmam, 1999).

O modelo da necessidade-preocupação é de facto útil para explicar a não adesão (Butler et al., 2004; Horne et al., 2001; Horne \& Weinmam, 1999, 2002; Neame \& Hammond, 2005; Ross et al., 2004). Byrne et al. (2005) verificaram que as crenças sobre a medicação estavam moderadamente relacionadas com a adesão à medicação, bem como que crenças fortes sobre a necessidade em tomar a medicação e poucas preocupações estavam associadas a elevados níveis de adesão. No presente estudo, as crenças sobre as preocupações acerca da medicação não se relacionaram significativamente com a adesão, não se confirmando assim a hipótese mas, por sua vez, as crenças sobre a necessidade da toma a medicação parecem funcionar como um potenciador da adesão terapêutica e o contrário também é verdade em relação aos efeitos nocivos da medicação.

No que diz respeito às diferenças de género, verificamos que as mulheres apresentam um maior número de crenças sobre a necessidade da medicação, bem como um maior número de preocupações comparando com os homens. De facto, a literatura sobre comportamentos de estilo de vida saudáveis, indica que as mulheres apresentam uma orientação para a saúde mais positiva do que os homens e adoptam com mais frequência, comportamentos de saúde (Brubaker, 2007). Pereira e Silva (2002) verificaram, ao estudar a comportamentos de saúde e adesão terapêutica em usuários de um centro saúde, que as mulheres tinham uma prática de comportamentos saudáveis superior à dos homens. Acreditar que a medicação é necessária pode significar conhecimento sobre a doença e tratamento, que não é só importante na adesão à medicação, mas também na adesão a outros comportamentos de autocuidados fundamentais para o controlo da diabetes. Sousa e McIntyre (2008), numa amostra de diabéticos tipo 2 , verificaram a importância dos conhecimentos e da informação na adesão a vários comportamentos de autocuidados, bem como o seu papel como preditor desses comportamentos. Contudo, os estudos relativos ao género e adesão não são consensuais e alguns apontam para que os homens adiram mais à medicação do que as mulheres (Gimenes, Zanetti, \& Haas, 2009; Hertz, Unger, \& Lustik, 2005).

Relativamente à idade, não encontramos diferenças quanto às crenças gerais e específicas sobre a medicação. Independentemente da idade, os diabéticos apresentam um sistema de crenças gerais e específicas muito semelhantes. Esse resultado pode estar relacionado com o facto de todos os diabéticos da amostra terem sido diagnosticados há menos de um ano.

Verificaram-se diferenças em relação ao género e idade quando as três escalas sobre crenças acerca da medicação e a adesão à medicação foram relacionadas. No que diz respeito ao género, verificou-se que as mulheres que aderem à medicação apresentam maior número de crenças sobre a necessidade da medicação o que vai ao encontro do modelo da "necessidade-preocupação" de Horne et al. (2001). Os homens que aderem à medicação apresentam menor núme- ro de crenças gerais sobre a medicação (toxicidade e uso excessivo) como seria de esperar, mas apresentam crenças sobre as preocupações específicas com a medicação. Assim, esse resultado de maior adesão não evita a preocupação com a medicação.

Independentemente da idade, ambos os grupos que aderem à medicação apresentam crenças sobre a necessidade da medicação. Esse resultado pode ser devido ao facto dos diabéticos mais jovens estarem mais capacitados e dotados de competências (Yen \& Moss, 1999) para reconhecerem o benefício da adesão e os mais velhos terem maior conhecimento sobre a importância da adesão, nas doenças crónicas, dada a sua experiência de vida.

\section{Conclusões}

A versão portuguesa da Parte I (Crenças Gerais) apresenta-se de forma diferente à proposta pelo autor. Assim, a Parte I é constituída apenas por um factor de oito itens e a Parte II (Crenças Específicas) dividiu-se em dois factores como o autor sugeriu: crenças específicas sobre a necessidade da medicação constituída por cinco itens e crenças específicas sobre a preocupação com a medicação também constituída por cinco itens. Por fim, essas três escalas apresentam uma elevada validade convergente, correlacionando-se de forma significativa com a adesão à medicação. A relação entre as crenças e a adesão é sensível ao género e à idade. De acordo com os resultados obtidos, o Questionário Crenças acerca da Medicação, na versão portuguesa, revelou-se um instrumento com boas características psicométricas, passível de ser utilizado com pacientes diabéticos tipo 2. Tendo em conta a relação entre as crenças e a adesão a medicação, é importante que as crenças dos pacientes sobre os medicamentos sejam avaliadas e incluídas em programas de intervenção na diabetes, no sentido de aumentar a adesão terapêutica, tendo em conta as diferenças de género.

Dada a importância das crenças sobre a medicação, a sua relação com a adesão terapêutica à medicação e o facto das crenças afectarem a forma e o conteúdo da comunicação acerca da medicação, por parte do profissional de saúde, seria importante avaliar o impacto da possível discrepância entre as crenças do doente e as das crenças do profissional de saúde, na adesão do doente a medicação, em estudos futuros. De facto, os profissionais de saúde podem assumir que os doentes possuem as mesmas crenças que eles próprios acerca da medicação e, como tal não abordarem esse aspecto nas suas consultas. Assim, seria importante que as crenças acerca da medicação fizessem parte duma decisão compartilhada entre profissional de saúde-doente (Weiss \& Britten, 2006) já que a concordância é fundamental na adesão terapêutica (Clifford, Barber, Elliott, Hartley, \& Horne, 2006; Stevenson, Cox, Britten, \& Dundar, 2004).

É relevante enfatizar a necessidade de os profissionais de saúde abordarem as crenças acerca da medicação de uma forma regular ao longo da doença, dado que o doente pode mudar as suas crenças em função da experiência que vai adquirindo com o tratamento em função da necessidade da medicação, preocupações, uso excessivo e efeitos nocivos. Por último, seria importante ter em consideração que são 
sobretudo as mulheres diabéticas que mais crenças sobre necessidades da medicação e sobre preocupações com a medicação apresentam, e ser, portanto, um grupo com mais necessidade de avaliação nesse nível.

\section{Referências}

Aikens, J. E., Nease, D. E. Jr., Nau, D. P., Klinkman, M. S., \& Schwenk, T. L. (2005). Adherence to maintenance-phase antidepressant medication as a function of patient beliefs about medication. Annals of Family Medicine, 3(1), 23-30.

Aikens, J. E., \& Piette, J. D. (2009). Diabetic patients' medication underuse, illness outcomes, and beliefs about antihyperglycemic and antihypertensive treatments. Diabetes Care, 32(1), 19-24.

Asefzadeh, S., Asefzadeh, M., \& Javadi, H. (2005). Care management: Adherence to therapies among patients at $\mathrm{Bu}-$ Alicina Clinic. Research Journal of Medicine and Medical Sciences, 10, 343-348.

Ayalon, L., Arean, P. A., \& Alvidrez, J. (2005). Adherence to antidepressant medications in black and Latino elderly patients. American Journal of Geriatric Psychiatry, 13(7), 572-580.

Barnes, L., Moss-Morris, R., \& Kaufusi, M. (2004). Illness beliefs and adherence in diabetes mellitus: A comparison between Tongan and European patients. The New Zealand Medical Journal, 117(1188), 1-9.

Benson, J., \& Britten, N. (2002). Patients' decisions about whether or not to take antihypertensive drugs: Qualitative study. British Medical Journal, 325(19), 1-5.

Bradley, C. (1996). Handbook of psychology and diabetes. A guide to psychological measurement, diabetes research and practice. London: Harwood Academic Publishers.

Britten, N. (1994). Patients' ideas about medicines: A qualitative study in a general practice population. British Journal of General Practice, 44, 465-468.

Brubaker, C. E. (2007, August). Gender-role orientation, family structure and health behavior: A focus on physical activity. Paper presented at the annual meeting of the American Sociological Association, New York.

Butler, J. A., Peveler, R. C., Roderick, P., Smith, P. W. F., Horne, R., \& Mason, J. C. (2004). Modifiable risk factors for non-adherence to immunosuppressants in renal transplant recipients: A cross sectional study. Nephrology Dialysis Transplantation, 19(12), 3144-3149.

Byrne, M., Walsh, J., \& Murphy, A. W. (2005). Secondary prevention of coronary heart disease: Patient beliefs and health-related behaviour. Journal of Psychosomatic Research, 58(5), 403-415.

Chapman, R. H., Petrilla, A. A., Benner, J. S., Schwartz, J. S., \& Tang, S. S. (2008). Predictors of adherence to concomitant antihypertensive and lipid-lowering medications in older adults: A retrospective, cohort study. Drugs Aging, 25(10), 885-892.

Clifford, S., Barber, N., Elliott, R., Hartley, E., \& Horne, R. (2006). Patient-centred advice is effective in improving adherence to medicines. Pharmacy World \& Science, 28, 165-170.

Clifford, S., Barber, N., \& Horne, R. (2008). Understanding different beliefs held by adherers, unintentional nonadherers, and intentional nonadherers: Application of the necessityconcerns framework. Journal of Psychosomatic Research, 64(1), 41-46.
Cramer, J. A. (2004). A systematic review of adherence with medications for diabetes. Diabetes Care, 27, 1218-1224.

Donovan, J. L., \& Blake, D. R. (1992). Patient noncompliance: Deviance or reasoned decision-making? Social Science and Medicine, 34(5), 507-513.

Dowell, J., \& Hudson, H. (1997). A qualitative study of medicationtaking behavior in primary care. Family Practice, 14(5), 369-375.

Duarte, R. (Ed.). (2004). Diabetelogia clínica. Lisboa: Lidel.

Elliott, R. A., Ross-Degnan, D., Adams, A. S., Safran, D. G., \& Soumerai, S. B. (2007). Strategies for coping in a complex world: Adherence behavior among older adults with chronic illness. Journal of General Internal Medicine, 22(6), 805-810.

Farmer, A., Kinmonth, A-L., \& Sutton, S. (2006). Measuring beliefs about taking hypoglycaemic medication among people with type 2 diabetes. Diabetes Medicine, 23, 265-270.

Ferreira, M. S. (2009). Variáveis psicológicas na Lombalgia Crónica: Um estudo com doentes em tratamento de fisioterapia e acupunctura. (Tese de Doutorado não- publicada). Universidade do Minho, Braga, Portugal.

Figueiras, M., Marcelino, D., Claudino, A., Cortes, M., Maroco, J., \& Weinman, J. (2009). Patients' illness schemata of hypertension: The role of beliefs for the choice of treatment. Psychology \& Health, 25(4), 507-517.

Gimenes, H. T., Zanetti, M. L., \& Haas, V. J. (2009). Factors related to patient adherence to anti diabetic drug therapy. Revista Latino-Americana de Enfermagem, 17(1), 46-51.

Hampson, S. E., Glasgow, R. E., \& Foster, L. S. (1995). Personal models of diabetes among older adults: Relationship to selfmanagement and other variables. Diabetes Education, 21, 300-307.

Hertz, R., Unger, A., \& Lustik, M. (2005). Adherence with pharmacotherapy for type 2 diabetes: A retrospective cohort study of adults with employer-sponsored health insurance. Clinical Therapeutics, 27(7), 1064-1073.

Horne, R. (1997). Representations of medication and treatment: Advances in theory and measurement. In K. J. Petrie \& J. A. Weinman (Eds.), Perceptions of health and illness (pp. 155188). Amsterdam: Harwood.

Horne, R. (1999). Patients' beliefs about treatment: The hidden determinant of treatment outcome? Journal of Psychosomatic Research, 47(6), 491-495.

Horne, R. (2001a). Compliance, adherence and concordance. In P. Gard (Eds.), Behavioural approach to pharmacy practice (pp. 166-183). London: Blackwell.

Horne, R. (2001b). Non-adherence to medication: Causes and implications for care. In P. Gard (Ed.), A behavioral approach to pharmacy practice (pp. 111-130). Oxford: Blackwell.

Horne, R. (2003). Treatment perceptions and self-regulation. In L.D. Cameron \& H. Leventhal (Eds.), The self-regulation of health and illness behavior (pp. 138-154). London: Routledge.

Horne, R. (2006). Beliefs and adherence to treatment. The challenge for research and practice. In P. Halligan \& M. Aylard (Eds.), The power of belief psychosocial influence on illness, disability and medicine (pp. 115-136). Oxford: Oxford University Press.

Horne, R., Buick, D., Fisher, M., Leake, H., Cooper, V., \& Weinman, J. (2004). Doubts about necessity and concerns about adverse effects: Identifying the types of beliefs that are associated with nonadherence to HAART. International Journal of STD \& AIDS, 15(1), 38-44. 
Horne, R., Frost, S., Hankins, M., \& Wright, S. (2001). In the eye of the beholder: Pharmacy students have more positive perceptions of medicines than students of other disciplines. International Journal of Pharmacy Practice, 9, 85-89.

Horne, R., \& Weinman, J. (1999). Patients' beliefs about prescribed medicines and their role in adherence to treatment in chronic physical illness. Journal of Psychosomatic Research, 47, 555-567.

Horne, R., \& Weinman, J. (2002). Self-regulation and selfmanagement in asthma: Exploring the role of illness perceptions and treatment beliefs in explaining non-adherence to preventive medication. Psychology and Health, 17, 17-32.

Horne, R., Weinman, J., \& Hankins, M. (1999). The beliefs about medicines questionnaire (BMQ): A new method for assessing cognitive representations of medication. Psychology and Health, 10, 1-29.

Kavanagh, D. J., Gooley, S., \& Wilson, P. H. (1993). Prediction of adherence and control in diabetes. Journal of Behavioral Medicine, 16, 509-522.

Lau, D. T., Briesacher, B., Mercaldo, N. D., Halpern, L., Osterberg, E. C., Jarzebowski, M....Mazor, K. (2008). Older patients' perceptions of medication importance and worth: An exploratory pilot study. Drugs Aging, 25(12), 1061-1075.

Lee, W. C., Balu, S., Cobden, D., Joshi, A. V., \& Pashos, C.L. (2006). Prevalence and economic consequences of medication adherence in diabetes: A systematic literature review. Managing Care Interface 19, 31-41.

Leventhal, H., Meyer, D., \& Nerenz, D. (1980). The common sense representation of illness danger. In S. Rachman (Ed.), Contributions to medical psychology (pp. 7-30). Oxford: Pergamon Press.

Mardby, A. C., Akerlind, I., \& Jorgensen, T. (2007). Beliefs about medicines and self-reported adherence among pharmacy clients. Patient Education Counseling, 69(1-3), 158-164.

McHorney, C., \& Gadkari, A. (2010). Individual patients hold different beliefs to prescription medications to which they persist VS nonpersist and persist VS nonfulfill. Patient Preference and Adherence, 4, 187-195.

Menckeberg, T. T., Bouvy, M, L., Bracke, M., Kaptein, A. A., Leufkens, H. G., Raaijmakers, J. A. M.,...Horne, R. (2008). Beliefs about medicines predict refill adherence to inhaled corticosteroids. Journal of Psychosomatic Research, 64(1), 47-54.

Nair, K. M., Levine, M. A., Lohfeld, L. H., \& Gerstein, H.C. (2007). "I take what I think works for me". A qualitative study to explore patient perception of diabetes treatment benefits and risks. Canadian Journal of Clinical Pharmacology, 14(2), 251-259.

Neame, R., \& Hammond, A. (2005). Beliefs about medications: A questionnaire survey of people with rheumatoid arthritis. Rheumatology, 44(6), 762-769.

Pereira, M. G., \& Silva, S. (2002). Atribuição de sintomas, comportamentos de saúde e adesão em utentes de centro de saúde da Zona Norte. Análise Psicológica, 1(XX), 35-43.

Phatak, H. M., \& Thomas, J. (2006). Relationships between beliefs about medications and nonadherence to prescribed chronic medications. The Annals of Pharmacotherapy, 40, 1737-42.
Ramston, H., Afandi, S., Elofsson, K., \& Petersson, S. (2009). Differences in beliefs patients and pharmaceutical specialists regarding medications. Patient Education and Counseling, 62, 244-249.

Reis, J. C. (2005). O que é a saúde? Significações pessoais, modelos científicos e educação para a saúde. Lisboa: Nova Veja.

Ross, S., Walker, A., \& MacLeod, M. J. (2004). Patient compliance in hypertension: The role of illness perceptions and treatment beliefs. Journal of Human Hypertension, 18, 607-613.

Sousa, M. R., \& McIntyre, T. (2008). Conhecimento do diabético sobre a doença e a repercussão no tratamento. Revista Brasileira em Promoção da Saúde, 21(4), 281-289.

Stevenson, F. A., Cox, K., Britten, N., \& Dundar, Y. (2004). A systematic review of the research on communication between patients and health care professionals about medicines: The consequences for concordance. Health Expect, 7, 235-245

Teixeira, J. A. C., \& Leal, I. (1993). Psicologia da saúde: Contexto e intervenção. Análise Psicológica, 8(4), 453-458.

Tibaldi, G., Clatworthy, J., Torchio, E., Argentero, P., Munizza, C., \& Horne, R. (2009). The utility of the necessity-concerns framework in explaining treatment non-adherence in four chronic illness groups in Italy. Chronic Illness, 5(2), 125-133.

Tordera, M. P., Moragon, E. M., Fuster, A. B., Bayo, A. L., \& Ciscar, C. P. (2009). Spanish asthma patients' beliefs about health and medicines: Validation of 2 questionnaires. Archives of Bronconeumologia, 45(5), 218-223.

Treharne, G. J., Lyons, A. C., \& Kitas, G. D. (2004). Medication adherence in rheumatoid arthritis, effects of psychological factors. Psychology of Health Medicine, 9, 337-349.

Vermeire, E., Hearnshaw, H., Van Royen, P., \& Denekens, J. (2001). Patient adherence to treatment: Three decades of research. A comprehensive review. Journal of Clinical Pharmacy and Therapeutics, 26, 331-342.

Weiss, M., \& Britten, N. (2003). What is concordance? Pharmaceutical Journal, 271, 493.

Williams, A. F., Manias, E., \& Walker, R. (2008). Adherence to multiple, prescribed medications in diabetic kidney disease: A qualitative study of consumers' and health professionals' perspectives. International Journal of Nursing Studies, 45(12), 1743-1756.

World Health Organization. (2003). Adherence to long term therapy: Evidence for action. Disponível em: ttp://www.who.int/chp/ knowledge/

Yen, I., \& Moss, N. (1999). Unbundling education: A critical discussion of what education confers and how it lowers risk for disease and death. Annals of the New York Academy of Sciences, 896, 350-351.

Recebido em 23.02.2012

Primeira decisão editorial em 02.05.2012

Versão final em 23.10.2012

Aceito em 29.10.2012 